\title{
A Face Recognition Technique using Local Binary Pattern Method
}

\author{
Sarabjit Singh ${ }^{1}$, Amritpal Kaur ${ }^{2}$, Taqdir ${ }^{3}$ \\ Student M.Tech (CSE), Computer Science, Guru Nanak Dev University RC, Gurdaspur, India ${ }^{1,2}$ \\ Assistant Professor, Computer Science, Guru Nanak Dev University RC, Gurdaspur, India ${ }^{3}$
}

\begin{abstract}
LBP is really a very powerful method to explain the texture and model of a digital image. Therefore it was ideal for feature extraction in face recognition systems. A face image is first split into small regions that LBP histograms are extracted and then concatenated in to a single feature vector. This vector forms an efficient representation of the face area and can be used to measure similarities between images. Automatic facial expression analysis is a fascinating and challenging problem, and impacts important applications in several areas such as humancomputer interaction and data-driven animation. Deriving a facial representation from original face images is an essential step for successful facial expression recognition method. In this paper, we evaluate facial representation predicated on statistical local features, Local Binary Patterns, for facial expression recognition. Various machine learning methods are systematically examined on several databases. Broad experiments illustrate that LBP features are effective and efficient for facial expression recognition.
\end{abstract}

Keywords: Face recognition, LBP histogram, Local Binary Patterns, Feature Extraction, LBP code.

\section{INTRODUCTION}

Face recognition is an active research area, and they can be utilized in wide range applications such as surveillance and security. Face is a sophisticated multidimensional structure and needs a good computing technique for recognition. Face recognition system can be utilized in two modes: Verification and Identification. Facial feature extraction includes localizing probably the most characteristics features of the face image like eyes, nose and mouth regions. There are two forms of solution to extract features from the facial images and they are:

Geometric feature considers the deviation fit, location, space between two eyes and period of the nose. The looks feature presents the looks variations of the face image, such as wrinkles and furrows. The looks feature needs to be extracted on either the whole face image or specific regions in a facial image. The face is certainly one of the main features of the human beings and usually used as identification. Recognizing different human faces is not an arduous task for humans, but it is quite hard to the system to acknowledge the human faces. LBP generate the binary code that describes local texture pattern by normalizing intensity values in neighborhood. The eyes and nose region is extracted from the LBP face image and then LBP histograms are drawn for each pixel of the image.PCA is just a way of identifying patterns in data and expressing the info to highlight their similarities and differences. [1,2]

\section{Image Acquisition}

This is actually the first faltering step where we acquire image using any digital device Images employed for facial expression recognition are static images or image sequences. 2-D monochrome (grey-scale) facial image sequences are the most popular form of pictures employed for automatic expression recognition.
Pre-processing

Image pre-processing takes the form of signal conditioning (such as noise removal, and normalization from the variation of pixel position or brightness), along with segmentation, location, or tracking of the face or its parts.

\section{Feature Extraction}

Feature extraction converts pixel data in to a higher-level representation of shape, motion, colour, texture, and spatial configuration of the facial skin or its components. Classification

Expression categorization is conducted by a classifier, which frequently contains types of pattern distribution, coupled to a choice procedure. A wide range of classifiers, covering parametric along with non-parametric techniques, has been placed on the automatic expression recognition problem.

Post-processing

Post-processing aims to boost recognition accuracy, by required domain knowledge to correct classification errors or by coupling together several degrees of a classification hierarchy.

\section{LOCAL BINARY PATTERN}

The area binary pattern (LBP) was originally designed for texture description. It's invariant to monotonic grey- scale transformations which are essential for texture description and analysis for the reason of computational simplicity processing of image in real-time is possible. With LBP it's possible to explain the texture and model of an electronic digital image. This is completed by dividing a picture into several small regions from which the features are extracted. These features contain binary patterns that describe the environmental surroundings of pixels in the regions. The features that are formed from the regions are concatenated into a single feature histogram, which describes to forms a representation of the image. Images 
will then be compared by measuring the similarity texture pattern is created by threshold a community by the (distance) between their histograms. According a number gray value of its center. The operator relates to many wellof studies face recognition utilising the LBP method known texture analysis methods.[7]

provides positive results, both with regards to speed and Designers of LBP face three fundamental problems: The discrimination performance. Due to the way the texture very first issue is how to describe different local patterns and model of images is described, the technique is of textures and then how exactly to extract these local apparently quite robust against face images with different patterns. Since not all of local patterns are with the same facial expressions, different lightening conditions, aging of importance to texture analysis. The second issue is how to persons and image rotation.
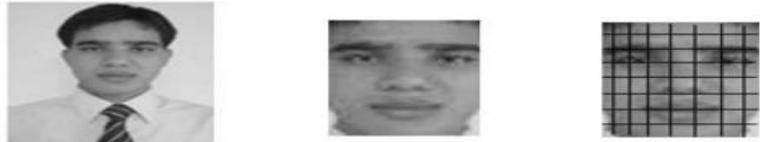

Fig. 1. A preprocessed image divided into 64 regions

Facial representation based on Local Binary Pattern (LBP) features for person-independent facial expression recognition. LBP features were proposed originally for texture analysis, and recently have now been introduced to represent faces in facial images analysis. The most crucial properties of LBP features are their tolerance against illumination changes and their computational simplicity. We examine different machine learning methods, that are template matching, Support Vector Machine (SVM), Linear Discriminant Analysis (LDA) and the linear programming technique, to execute facial expression recognition using LBP features [3][5][6].

Local binary patterns were introduced as a fine scale texture descriptor. An LBP description of a pixel is developed by thresholding the values of the $3 \times 3$ neighborhood of the pixel against the central pixel and interpreting the result as a binary number.

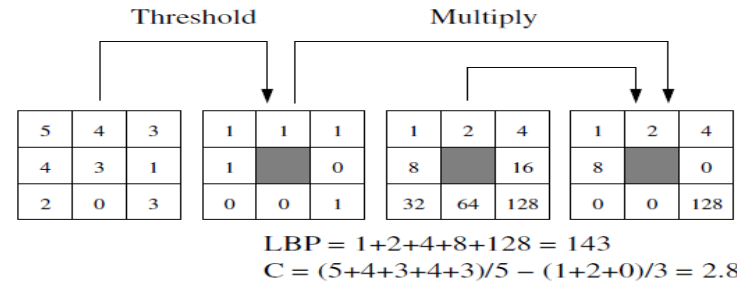

Fig. 2. Calculating the original LBP code and a contrast measure.
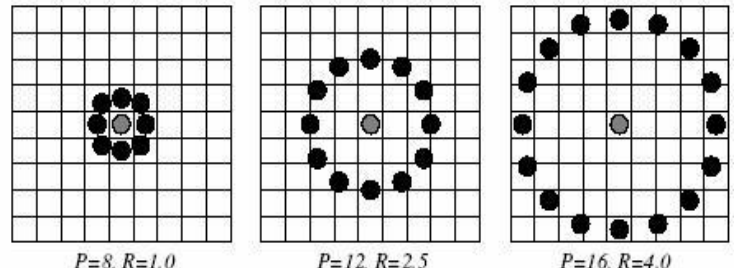

Fig. 3. Circularly symmetric neighbour sets

With the LBP operator, good discrimination rates were reported with textures selected from the photographic album. The present form of the LBP operator, described in Fig 2, is fairly different from this basic version: the original definition is extended to arbitrary circular neighbourhoods, and various types of extensions have already been developed. The basic idea behind this is however the same: a binary code that describes the local pick the primary subset of these local patterns to represent textures. The third issue is how to utilize these selected local patterns to form a fruitful texture descriptor [4][5].

The Local Binary Pattern (LBP) texture analysis operator is defined as a gray-scale invariant texture measure, based on a general definition of texture in an area neighborhood. The existing type of the LBP operator is very distinctive from its basic version: the initial definition is extended to arbitrary circular neighbourhoods, and various types of extensions have been developed. The fundamental idea is however the exact same: a binary code that describes the neighborhood texture pattern is built by thresholding a neighborhood by the gray value of its center.

Through its recent extensions, the LBP operator has been made into a very powerful measure of image texture, showing positive results in terms of accuracy and computational complexity. The LBP operator can be seen as a unifying way of the traditionally divergent statistical and structural models of texture analysis classifications [8][9].

The LBP feature vector, in its simplest form, is created in this manner:

Divide the examined window to cells (e.g. 16x16 pixels for each cell).

For every single pixel in the cell, compare the pixel to each of the 8 neighbors (on its left top, left middle, left bottom, right top, etc.). Follow the pixels along the circle, i.e. clockwise or counter clockwise direction.

Where the center pixel's value is greater compared to neighbour, write "1 ".Otherwise, write "0 ".This gives an 8 -digit binary number (which is generally transformed into decimal for convenience)

Compute the histogram, on the cell, of the frequency of each "number" occurring (i.e., each combination of which pixels are smaller and which are greater compared to center).

Optionally normalize the histogram.

Concatenate normalized histograms of most cells. This gives the feature vector for the window.

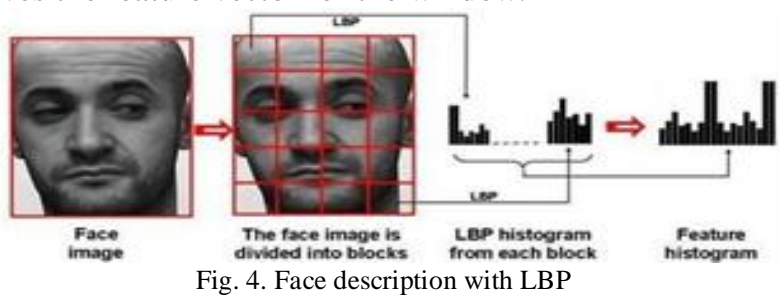

The derivation of the LBP follows that represented by Ojala. Let's define texture $T$ whilst the joint distribution of the gray quantities of $P+1(\mathrm{P}>0)$ image pixels:

$T=t\left(g_{c}, g_{0}, \ldots, g_{p-1}\right)$, 
where $g_{c}$ corresponds to the gray value of the center pixel classification phase. Although local binary features has of a local neighborhood. $g_{p}(p=0, \ldots, P-1)$ match the gray been extracted from the face image for face recognition values of $P$ equally spaced pixels on a group of that there are several face image uses in the database that radius $R(R>0)$ that form a circularly symmetric pair of compared with the input face image. The face image neighbours[3,4]. Figure 4 illustrates three circularly depends on viewing lighting and environmental symmetric neighbour sets for different values conditions. In addition the face image changes according of $P$ and $R$. Without losing information, $g_{c}$ could be to the expressions. In the research work, which is flexible subtracted from $g_{p}$ :

$T=t\left(g_{c}, g_{0^{-}} g_{c}, \ldots, g_{p-1^{-}} g_{c}\right)$.

Assuming that the differences are independent of $g_{c}$, the distribution could be factorized:

$T=t\left(g_{c}\right) t\left(g_{0^{-}} g_{c}, \ldots, g_{p-1}-g_{c}\right)$.

Since $t\left(g_{c}\right)$ describes the general luminance of a graphic, which is unrelated to local image texture, it may be ignored:

$T=t\left(g_{0}-g_{c}, \ldots, g_{p-1^{-}} g_{c}\right)$.

Although invariant against gray scale shifts, the differences are influenced by scaling. To attain invariance with respect to any monotonic transformation of the gray scale, only the signs of the differences are taken in this method:

$T=t\left(s\left(g_{0^{-}} g_{c}\right), \ldots, s\left(g_{p-1}-g_{c}\right)\right)$,

Now, a binomial weight $2^{p}$ is assigned to each sign $s\left(g_{p}\right.$ $g_{c}$ ), transforming the differences in a neighborhood into a unique LBP code:

$$
\operatorname{LBP}_{P . R}\left(x_{c}, y_{c}\right)=\sum_{p=0}^{p-1} s\left(g_{p}-g_{c}\right)^{p}
$$

The first LBP is described by two ways. First, the neighborhood in the typical definition is indexed circularly, rendering it better to derive rotation invariant texture descriptors. Second, the diagonal pixels in the $3 \times 3$ neighborhood are interpolated in LBP. [10,11,12]

\section{Algorithm}

We start by summarizing the main common steps of the algorithms used in this work. Then we describe each step in detail. The proposed face recognition process consists of four main parts:

1) Preprocessing: We begin by applying the Tan and Triggs' illumination normalization algorithm to compensate for illumination variation in the face image. No further preprocessing, such as for example face alignment, is performed in the preprocessing.

2) LBP operator application: In the 2 nd stage LBP are computed for each pixel, making a fine scale textural description of the image.

3) Local feature extraction process: Local features are produced by computing histograms of LBP over local image regions.

4) Classification: Each face image in test set is classified by comparing it against the face images in the training set. The comparison is performed using the local features obtained in the previous step in the algorithm.

The first two steps are shared by all the algorithms. The algorithms we explore in this work vary in how they perform the last two steps. [7]

Face recognition is not a simple problem since an unknown face image seen in the extraction phase is usually different from the face image seen in the and efficient, should be solved the desired problems.

[12-15]To implement the face recognition in this research work, we proposed the Local Binary patterns methodology. Local Binary Pattern works on local features that uses LBP operator which summarizes the local special structure of a face image. [8]

LBP is defined as an orders set of binary comparisons of pixels intensities between the center pixels and its eight surrounding pixels in the image.
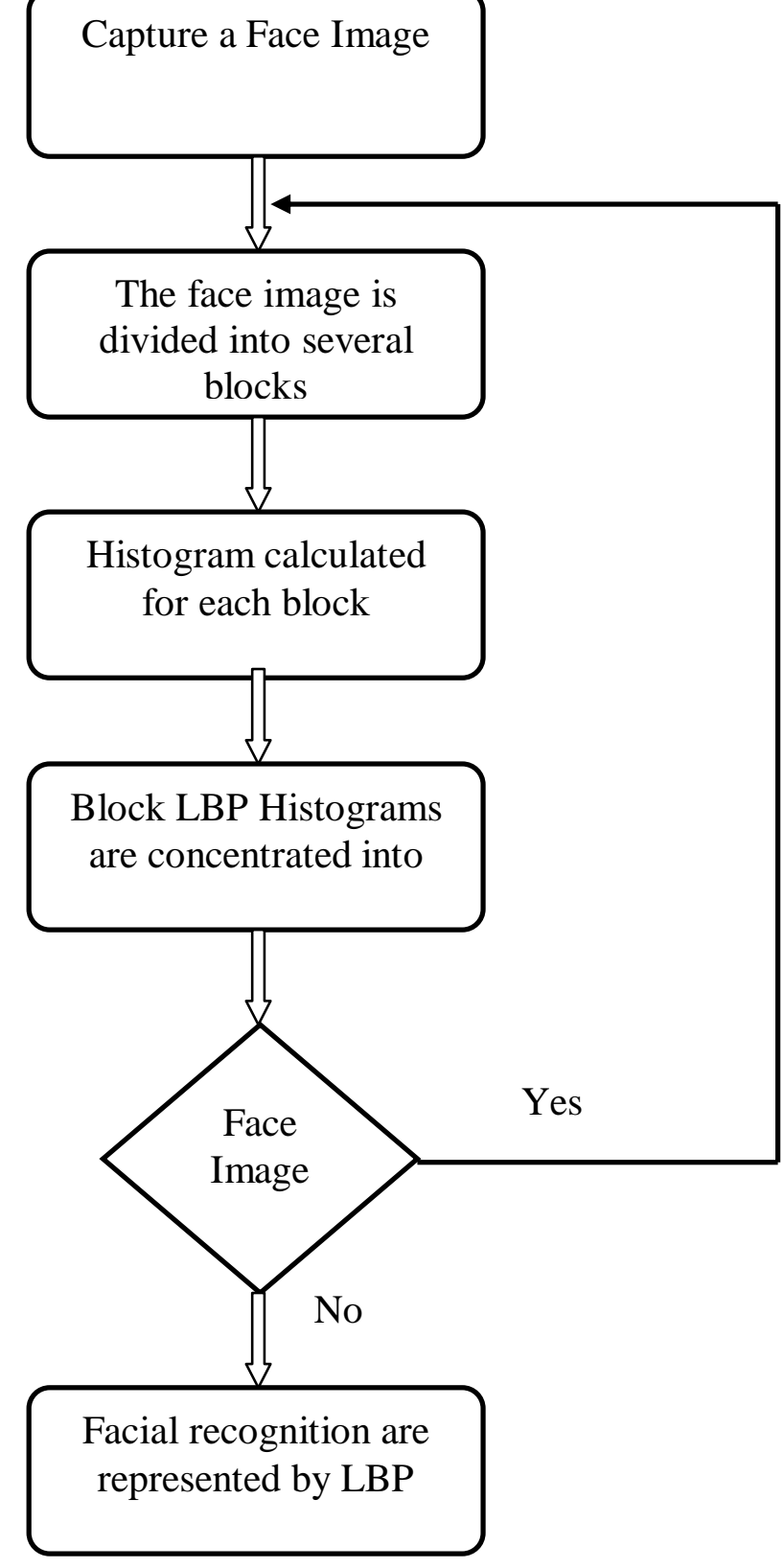

Fig. 5. Flow Chart for Local Binary Pattern 


\section{III.CONCLUSION}

In this paper, a survey on local binary pattern for face recognition has been studied in detail. Face recognition has become a well known topic in various applications like security, surveillance etc. In LBP the entire image is split into equal sized blocks. Then local binary pattern is computed for each pixel in most blocks by comparing the guts pixel with neighbouring pixels. This may give us a binary pattern for each image.

\section{ACKNOWLEDGMENT}

We sincerely acknowledge the immense contribution of Er. Taqdir (Assistant Professor, Department of Computer Science and Engineering, Guru Nanak Dev University RC, Gurdaspur) for her guidance, encouragement and supervision during the period of this work.

\section{REFERENCES}

[1] T. Ojala and M. Pietikainen. Multiresolution Gray-Scale and Rotation Invariant Texture Classification with Local Binary Patterns, IEEE Trans on Pattern Analysis and Machine Intelligence, Vol. 24. No.7, July, 2002.

[2] Ahonen, T., Hadid, A., Pietikäinen, M.: Face description with local binary patterns: Application to face recognition. IEEE Trans. Pattern Anal. Mach. Intell. 28(12), 2037-2041 (2006).

[3] AnaghaV.Malkapurkar, Rupali Patil, Prof. Sachin Murarka, A New Technique for LBP Method to Improve Face Recognition, International Journal of Emerging Technology and Advanced Engineering ISSN 2250-2459, Volume 1, Issue 1, November 2011.

[4] Ahonen, T., Hadid, A., Pietikäinen, M.: Face Recognition with Local Binary Patterns. In: Computer Vision, ECCV 2004 Proceedings, Lecture Notes in Computer Science 3021 (2004) 469-481.

[5] Caifeng Shan, Shaogang Gong, Peter W. McOwan Facial expression recognition based on Local Binary Patterns: A comprehensive study. Image and Vision Computing 27 (2009) 803-816

[6] C. Shan, S. Gong, P.W. McOwan, Robust facial expression recognition using local binary patterns, in: IEEE International Conference on Image Processing (ICIP), Genoa, vol. 2, 2005, pp. 370-373.

[7] Li Liu, Lingjun Zhao, Yunli Long, Gangyao Kuang, Paul Fieguth, Extended local binary patterns for texture classification : Image and Vision Computing 30 (2012) 86-99.

[8] Md. Abdur Rahim, Md. Najmul Hossain, Tanzillah Wahid \& Md. Shafiul Azam: Face Recognition using Local Binary Patterns (LBP), Global Journal of Computer Science and Technology Graphics \& Vision, Volume 13 Issue 4 Version 1.0 Year 2013.

[9] Bo Yang, SongcanChen, A comparative study on local binary pattern (LBP) based face recognition: LBP histogram versus LBP image: Neurocomputing120(2013)365-379.

[10] Timo Ahonen, Abdenour Hadid, and Matti Pietik“ainen, Face Recognition with Local Binary Patterns, Machine Vision Group, Pattern Analysis and Machine Intelligence 24 (2002) 971-987.

[11] Chetan Ballur \& Shylaja's “Application of Local Binary Pattern and Principal Component Analysis for Face Recognition"International Journal of Electrical, Electronics and Data communication, issn (p): 2320-2084, volume-1, issue-, july-2013.

[12] Fabrice Bourel \& C.C. Chibelushi's "Facial Expression Recognition:A Brief tutorial Overview" , School of Computing, Staffordshire University, Beaconside, Stafford ST18 ODG, ORSYP, 101 quartier Boieldieu, La Défense 8, F-92042 Paris La Défense Cedex, France.

[13] Taranpreet Singh Ruprah's "Face Recognition Based on PCA Algorithm", Special Issue of International Journal of Computer Science \& Informatics (IJCSI), ISSN (PRINT) : 2231-5292, Vol.- II, Issue-1, 2.

[14] Suman Kumar Bhattacharyya \& Kumar Rahul's "Face Recognition by Linear Discriminant Analysis", International Journal of Communication Network Security, ISSN: 2231 - 1882, Volume-2, Issue-2, 2013.

[15] S'ebastien Marcel, Yann Rodriguez and Guillaume Heusch's “On the Recent Use of Local Binary Patterns for Face Authentication", International Journal of Image and Video Processing, special issue on Facial Image Processing, Manuscript received May 1, 2006; revised October 20, 2006; accepted May 25, 2007. 